\title{
A Malignant Complication of an Esophagus Duplication Tardily Diagnosed
}

Louise $L^{1,2}$, Damien $\mathbf{B}^{1,2,3 *}$, Elodie $\mathbf{G}^{1,2}$, Michel $\mathbf{R}^{1,2}$, Sulpice $L^{1,2}$ and Bernard $M^{1,2}$

${ }^{1}$ Department of Hepatobiliary and Digestive Surgery, Pontchaillou Hospital, France

${ }^{2}$ University of Rennes, France

3INRA, UR1341ADNC, France

*Corresponding author: Bergeat Damien, Department of Hepatobiliary and Digestive Surgery, Pontchaillou Hospital, University Hospital Center, University of Rennes, France

Received: December 20, 2018; Accepted: J anuary 08, 2019; Published: J anuary 15, 2019

\section{Introduction}

Several theories exist to explain the embryologic development of those duplications, the omphalomesenteric remnant theory, the abortive twinning theory, the persistent embryologic diverticula theory, the split notochord theory, the aberrant luminal recanalization theory and the intrauterine vascular accidents theory $[1,2]$.

Most of the time, patients are diagnosed during childhood (70$90 \%$ before the age of two) [3]. During adulthood, complication is the most common situation to reveal this type of malformation.

Here, we report the rare case of a complete eso gastro duodenal duplication with a tumor evolution.

\section{Case Presentation}

A 25 years old female presented with epigastric pain relieved by vomiting, in a peripheral hospital. Her medical history was only marked by an appendectomy by laparotomy in the childhood.

After physical examination and classical laboratory tests, a CT scan was performed and visualized an intestinal malrotation with Ladd's bands.

An exploratory laparoscopy was performed and confirmed the incompleted common mesentery. The section of the Ladd's band was made. During the procedure, a gastro-duodenal duplication was revealed and a fibroscopy was performed during the operation to confirm this anomaly (Figure 1A). The procedure was stopped to explore the duplication. A CT-scan (Figure 1B) and an abdominal MRI completed the examination. A communication between the gastro duodenal duplication and the stomach and the duodenum was found. A resection of the gastric duplication was performed by laparotomy. Anatomopathological analysis showed fundic mucosal $29 \mathrm{~cm}$ length with duodenal metaplasia followed by duodenal mucosal $5,5 \mathrm{~cm}$ length. It showed no dysplasia or tumour site. No complications have occurred during the postoperative follow-up.

Four years later, she was admitted in our center for dysphagia, fever and basicervical pains. A CT scan and a MRI revealed a mediastinal and cervical retro thyroid collection and an esophageal duplication without any criteria for malignant transformation (Figure 2). No communication with the native esophagus and the esophageal mucocele duplication was identified. A surgical treatment was then indicated with the intent to mucocele duplication resection. The surgery was conducted in lateral thoracoscopy. Unfortunately, because of an important tension in the mucocele cavity, the duplication was open and a purulent fluid was evacuated. The extemporaneous examination of the thickening of the duplication wall was not in favor of any tumoral process. Finally, the anatomopathologic examination retained an invasive squamous cell carcinoma, well differentiated, with keratinization, staged pT2.

The multidisciplinary oncology staff decided a surveillance without adjuvant treatment.

Three months later, a subcutaneous nodule appeared at the orifice of the thoracoscopy trocar. A local recurrence was suspected. To treat this recurrence, amonobloc resection with the overlying and underlying ribs by an open approach was performed. The anatomopathologic results concluded to an invasive squamous cell carcinomaa which invaded striated muscle.

Subsequently, the patient received adjuvant chemotherapy (FOLFOX).

During this treatment, after 6 cures, a celiac lymphadenopathy appeared, which regressed after the six next cures. The treatment was completed by radiotherapy. Unfortunately, the disease kept evolving, with abdominal pains, chronic cough, weight lost and profound alteration of the general state. She died one year after the last surgery.

\section{Discussion}

We present here a rare case of a tubular gastro-duodenal duplication associated with a cystic esophageal duplication. This case was instructive by its rarity and its complexity. It has been previously reported that $7 \%$ of gastrointestinal tracts are associated with another duplication anywhere else on the gastro intestinal tract [2]. Thus, a complete evaluation looking for another intestinal tract duplication 

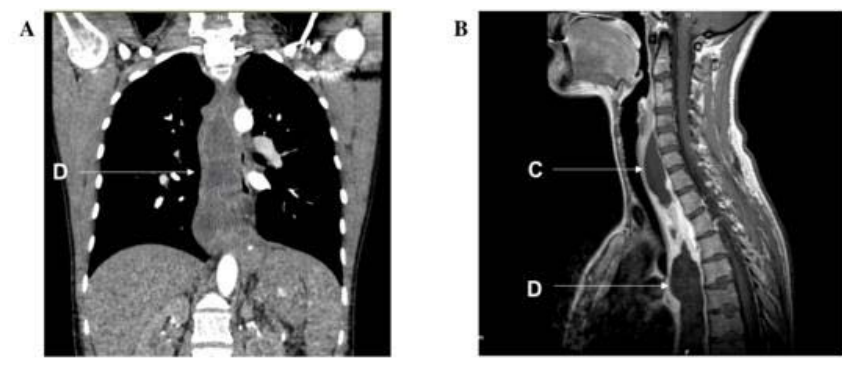

Figure 1: A CT scan and a MRI revealed a mediastinal and cervical retro thyroid collection and an esophageal duplication without any criteria for malignant transformation.
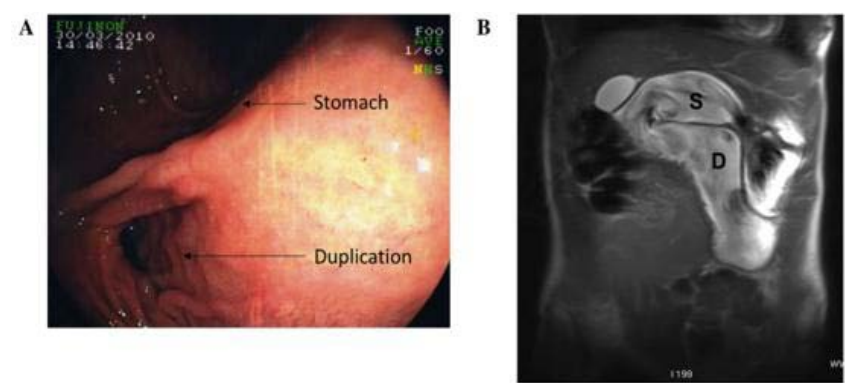

Figure 2: The section of the Ladd's band was made. During the procedure a gastro-duodenal duplication was revealed and a fibroscopy was performed during the operation to confirm this anomaly.

should have been performed by making a chest-abdomen-pelvis CT scan before any surgery.

Surgery is the main treatment of intestinal tract duplication. Several approaches are proposed in the literature. Some authors propose the possibility of a conservative treatment by performing a gastrogastrostomy by excising or obliterating the shared wall between the normal and duplicated stomach $[4,5]$. But, the risk of malignant evolution for alimentary tract duplication exists [6-8] and should not be neglected. Specifically, esophageal duplication cysts constitute between 0.5 and $2.5 \%$ of all esophageal tumors [9]. Furthermore, the literature suggests that esophagectomy and gastric interposition should be the treatment for tubular esophagus duplication even when there is no malignant transformation [10]. For esophageal duplication cyst, the resection of the cystic duplication is mandatory [11]. In case of a malignant transformation of the duplication cyst, we can wonder about the interest of a total resection of the cyst and the native esophagus. Indeed, the duplication shares a common blood and lymphatic supply with the native organ. For the same reasons, a complete lymph node dissection could be associated with the duplication cyst surgery. Our patient quickly developed a coeliac node after the surgery. If the initial oncologic transformation would had been suspected she probably could had beneficiated from an abdominal and a mediastinal lymphadenectomy during the initial surgery. Currently there is no recommendations about the place of a systematic lymph node dissection in the treatment of alimentary tract duplication in the absence of suspected malignant transformation.

We presented here a very rare case of a gastro duodenal duplication associated with a malignant transformed esophagus duplication. This case presented here is instructive by the succession of mistakes during the management. In case of intestinal duplication, an esophagus duplication has to be search and the potential malignant transformation of the duplication must be an obsession even if no clear recommendations are actually available concerning the surgical strategy.

\section{References}

1. Abdulla MAM, Al Saeed M, Ameer Alshaikh S, Nabar UJ. Adenocarcinoma arising from a gastric duplication cyst: a case report and literature review. Int Med Case Rep J. 2017;10:367-372.

2. Macpherson RI. Gastrointestinal tract duplications: clinical, pathologic, etiologic, and radiologic considerations. Radiogr Rev PublRadiol Soc N Am Inc. 1993;13:1063-1080.

3. Stern LE, Warner BW. Gastrointestinal duplications. SeminPediatr Surg. 2000;9:135-140.

4. Continuous communicating esophageal and gastric duplication - Journal of Pediatric Surgery. 2017.

5. Izzidien al-Samarrai AY, Crankson SJ, Sadiq S. The use of mechanical sutures in the treatment of gastric duplications. Z Kinderchir Organ Dtsch Schweiz Osterreichischen Ges Kinderchir Surg Infancy Child. 1989;44:186187.

6. Boivin Y, Cholette JP, Lefebvre R. Accessory esophagus complicated by an adenocarcinoma. Can Med Assoc J. 1964;90:1414-1417.

7. Seeliger B, Piardi T, Marzano E, Mutter D, Marescaux J, Pessaux P. Duodenal duplication cyst: a potentially malignant disease. Ann SurgOncol. 2012;19:3753-3754.

8. Dai Z-J, Kang H-F, Lin S, Bai M-H, Ma L, Min W-L, et al. Esophageal cancer with esophageal duplication cyst. Ann Thorac Surg. 2013;96:e15-16.

9. Kolomainen D, Hurley PR, Ebbs SR. Esophageal duplication cyst: case report and review of the literature. Dis Esophagus. 2017;11:62-65.

10. Saha AK, Kundu AK. Tubular duplication of the oesophagus presenting with dysphagia. Singapore Med J. 2014;55:e90-92.

11. Holcomb GW, Gheissari A, O'Neill JA, Shorter NA, Bishop HC. Surgical management of alimentary tract duplications. Ann Surg. 1989;209:167-174.
Austin J Surg - Volume 6 Issue 2 - 2019

ISSN : 2381-9030 | www.austinpublishing group.com

Damien et al. (C) All rights are reserved
Citation: Louise L, Damien B, Elodie G, Michel R, Sulpice L and Bernard M. A Malignant Complication of an Esophagus Duplication Tardily Diagnosed. Austin J Surg. 2019; 6(2): 1160. 